\title{
Introductory Clifford analysis
}

\author{
H. De Schepper \& F. Sommen \\ Clifford Research Group, Faculty of Engineering and Architecture, Ghent University \\ Building S22, Galglaan 2, B-9000 Gent, Belgium
}

\begin{abstract}
In this chapter an introduction is given to Clifford analysis and the underlying Clifford algebras. The functions under consideration are defined on Euclidean space and take values in the universal real or complex Clifford algebra, the structure and properties of which are also recalled in detail. The function theory is centered around the notion of a monogenic function, which is a null solution of a generalized Cauchy-Riemann operator, which is rotation invariant and factorizes the Laplace operator. In this way, Clifford analysis may be considered both as a generalization to higher dimension of the theory of holomorphic functions in the complex plane and a refinement of classical harmonic analysis. A notion of monogenicity may also be associated to the vectorial part of the Cauchy-Riemann operator, which is called the Dirac operator; some attention is paid to the intimate relation between both notions. Since a product of monogenic functions is, in general, no longer monogenic, it is crucial to possess some tools for generating monogenic functions: such tools are provided by Fueter's theorem on the one hand and the Cauchy-Kovalevskaya extension theorem on the other. A corner stone in this function theory is the Cauchy integral formula for representation of a monogenic function in the interior of its domain of monogenicity. Starting from this representation formula and related integral formulae, it is possible to consider integral transforms such as Cauchy, Hilbert and Radon transforms, which are important both within the theoretical framework and in view of possible applications.
\end{abstract}

Key words: Clifford algebra, monogenic function, Cauchy-Riemann operator, Cauchy's formula, integral transforms

\section{Introduction}

In a paper which was published posthumously, William Kingdon Clifford [Clifford, 1882] defined the algebra which later was named after him, as a generalization of both Grassmann's exterior algebra and Hamilton's quaternions. His aim was to create a structure encompassing the geometric and the algebraic properties of Euclidean space, whence he called his algebra a "geometric algebra". This name is justified since the universal Clifford algebra is the minimal extension of the $m$-dimensional Euclidean space to an associative algebra, carrying its geometric, algebraic and metric properties. The importance of these algebra's lies in the fact that they incorporate inside one single structure both the inner product and the wedge product (also called exterior or Grassmann product) of vectors. Classical examples of Clifford algebras are the real numbers, the complex numbers and the quaternions. Also the $\gamma$-matrices introduced by Paul Dirac in order to linearize the Klein-Gordon equation, are in fact generators for a particular Clifford algebra.

It was also Paul Dirac who, in his famous paper of 1928 about the spinning electron [Dirac, 1928], introduced the first "Dirac operator" as a "square root" of the wave operator in Minkowski space. 
This definition was generalized by Brauer and Weyl in 1935 [Brauer and Weyl, 1935] to any finite dimensional quadratic space with arbitrary signature.

In particular, constructing the universal Clifford algebra over $\mathbb{R}$, one obtains the twodimensional algebra of the complex numbers. In this framework one considers the Cauchy-Riemann operator, which underlies the theory of holomorphic functions in the complex plane, as consisting of a real and a vectorial part. Multiplying this operator with its complex conjugate, one obtains the twodimensional Laplacian. Holomorphic functions of one complex variable thus are null solutions of a first order elliptic differential operator, which factorises the Laplacian and may moreover be shown to be invariant under rotations.

By means of the universal Clifford algebra, this setting may be generalized in a very natural way to higher dimension by introducing a generalized Cauchy-Riemann operator containing a scalar and an $m$-dimensional vectorial part, which factorizes the $(m+1)$-dimensional Laplacian. The null solutions of this operator are generalized holomorphic functions, more commonly called monogenic functions. A notion of monogenicity may also be defined with respect to the $m$-dimensional vectorial operator, which is called the Dirac operator and factorizes the $m$-dimensional Laplacian.

A first contribution to the study of monogenic functions can be found in the work of R. Fueter, [Fueter, 1935a]. A detailed study, constituting the foundations of the current field, is the book by F. Brackx, R. Delanghe and F. Sommen, [Brackx et al, 1982]. Other standards are the books by Gilbert and Murray, [Gilbert and Murray, 1991], Gürlebeck and Sprössig, [Gürlebeck and Sprössig], and Gürlebeck, Habetha and Sprössig, [Gürlebeck et al, 2008]. In [Colombo et al, 2004], the authors complement and enrich the analytic approach of the Dirac system by the use of computers for discovering results in particular cases and for the application of Gröbner bases as a theoretical tool. Interesting basic references also are a thorough introduction by J. Ryan, [Ryan,2000], and an extensive overview paper by R. Delanghe, [Delanghe, 2001].

\section{Clifford algebras}

\subsection{Real Clifford algebras}

Let $m \in \mathbb{N}$ and let $\mathbb{R}^{0, m}$ be the real vector space $\mathbb{R}^{m}$ equipped with a non-degenerate symmetric bilinear form $\mathcal{B}$ of signature $(0, m)$, with associated quadratic form $\mathcal{Q}$. It means that, choosing an orthonormal basis $e=\left(e_{1}, \ldots, e_{m}\right)$ of $\mathbb{R}^{0, m}$ w.r.t. $\mathcal{B}$, one has that

$$
\mathcal{B}\left(e_{i}, e_{j}\right)=-\delta_{i j}, \quad i, j=1, \ldots, m
$$

whence in particular $\mathcal{Q}\left(e_{j}\right)=-1, j=1, \ldots, m$. Once the orthormal basis $e$ is chosen, one denotes an arbitrary vector $\underline{x} \in \mathbb{R}^{0, m}$ by $\underline{x}=\sum_{j=1}^{m} x_{j} e_{j}$. It then holds that

$$
\mathcal{B}(\underline{x}, \underline{y})=-\sum_{j=1}^{m} x_{j} y_{j} \quad \text { and } \quad \mathcal{Q}(\underline{x})=-\sum_{j=1}^{m} x_{j}^{2}=-|\underline{x}|^{2}
$$

where $|\underline{x}|$ stands for the Euclidean norm of $\underline{x}$.

The real Clifford algebra with generators $\left(e_{1}, \ldots, e_{m}\right)$ is a real linear associative algebra with identity 1 , containing $\mathbb{R}$ and $\mathbb{R}^{m}$ as subspaces. It is obtained by imposing the condition that for each vector $\underline{x}$ there should hold that $\underline{x}^{2}=\mathcal{Q}(\underline{x})=-|\underline{x}|^{2}$, whence the following multiplication rules are obtained for the generators:

$$
e_{i} e_{j}+e_{j} e_{i}=-2 \delta_{i j}, \quad i, j=1, \ldots, m
$$


or more explicitly

$$
\begin{aligned}
e_{i}^{2} & =-1, & i=1, \ldots, m \\
e_{i} e_{j}+e_{j} e_{i} & =0, & i \neq j, i, j=1, \ldots, m
\end{aligned}
$$

In view of these relations, every product of basis vectors $e_{\beta_{1}} \ldots e_{\beta_{s}}$, with $\beta_{1}, \ldots, \beta_{s} \in\{1, \ldots, m\}$, can be rewritten as $\pm e_{\alpha_{1}} \ldots e_{\alpha_{h}}$, with $1 \leq \alpha_{1}<\ldots<\alpha_{h} \leq m, h \leq m$. A basis for the Clifford algebra $\mathbb{R}_{0, m} \equiv \operatorname{Alg}_{\mathbb{R}}\left(e_{1}, \ldots, e_{m}\right)$ thus is given by the elements

$$
e_{A}=e_{\alpha_{1}} \ldots e_{\alpha_{h}}, \quad A=\left(\alpha_{1}, \ldots, \alpha_{h}\right), 1 \leq \alpha_{1}<\ldots<\alpha_{h} \leq m, h \leq m
$$

whence the algebra is $2^{m}$ dimensional. The identity element is obtained for $A=\emptyset$, i.e. $e_{\emptyset}=1$. An arbitrary Clifford number $a \in \mathbb{R}_{0, m}$ may thus be written as

$$
a=\sum_{A} a_{A} e_{A}, \quad a_{A} \in \mathbb{R}
$$

the sum being taken over all ordered subsets $A$ of $M=(1, \ldots, m)$.

A more abstract definition of the Clifford algebra can be found in the work of C. Chevalley, [Chevalley, 1997], as a factor space of a tensor algebra.

Example 1. Let $m=1$. The 2-dimensional Clifford algebra $\mathbb{R}_{0,1}$ generated by $e=\left(e_{1}\right)$ has the basis $\left(1, e_{1}\right)$, with $e_{1}^{2}=-1$. Identifying $e_{1}$ with the imaginary unit $i$, one has that $\mathbb{R}_{0,1} \simeq \mathbb{C}$.

Example 2. Let $m=2$. The 4-dimensional Clifford algebra $\mathbb{R}_{0,2}$ generated by $e=\left(e_{1}, e_{2}\right)$ has the basis $\left(1, e_{1}, e_{2}, e_{12} \equiv e_{1} e_{2}\right)$, with $e_{1}^{2}=e_{2}^{2}=-1$, and, on account of the multiplication rules, also $e_{12}^{2}=\left(e_{1} e_{2}\right)^{2}=-1$. Making the identifications $i=e_{1}, j=e_{2}$ and $k=e_{1} e_{2}$, one has that $\mathbb{R}_{0,2} \simeq \mathbb{H}$.

One may observe in $\mathbb{R}_{0, m}$ a so-called multivector structure. Indeed, rewriting the above form of the Clifford number $a$ as

$$
a=\sum_{A} a_{A} e_{A}=\sum_{k=0}^{m} \sum_{|A|=k} a_{A} e_{A}, \quad a_{A} \in \mathbb{R}
$$

it is readily seen that $\mathbb{R}_{0, m}$ decomposes as

$$
\mathbb{R}_{0, m}=\mathbb{R}_{0, m}^{0} \oplus \mathbb{R}_{0, m}^{1} \oplus \ldots \oplus \mathbb{R}_{0, m}^{m}
$$

where $\mathbb{R}_{0, m}^{k}$ is the space of so-called $k$-vectors in $\mathbb{R}_{0, m}$, given by

$$
\mathbb{R}_{0, m}^{k}=\operatorname{span}_{\mathbb{R}}\left\{e_{A} \mid A=\left(\alpha_{1}, \ldots, \alpha_{k}\right), 1 \leq \alpha_{1}<\ldots<\alpha_{k} \leq m\right\}
$$

The element $a$ may thus still be rewritten as $a=[a]_{0}+[a]_{1}+\ldots+[a]_{m}$, where $[a]_{k}$ represents the projection of $a$ on the space of $k$-vectors.

In particular, $\mathbb{R}_{0, m}^{0} \simeq \mathbb{R}$ are the scalars, $\mathbb{R}_{0, m}^{1} \simeq \mathbb{R}^{m}$ are the vectors, and $\mathbb{R}_{m}^{m} \simeq \mathbb{R} e_{M}$ is called the space of pseudoscalars. An important role is played also by the space of bivectors, i.e.

$$
\mathbb{R}_{0, m}^{2}=\operatorname{span}_{\mathbb{R}}\left\{e_{i} e_{j} \mid i<j\right\}
$$

since the Clifford product of two arbitrary vectors $\underline{x}$ and $\underline{y}$ decomposes as $\underline{x} \underline{y}=\underline{x} \bullet \underline{y}+\underline{x} \wedge \underline{y}$ into a scalar part

$$
\underline{x} \bullet \underline{y}=-\sum_{j=1}^{m} x_{j} y_{j}=-\langle\underline{x}, \underline{y}\rangle=\frac{1}{2}(\underline{x} \underline{y}+\underline{y} \underline{x})
$$


being, up to a minus sign, the standard Euclidean inner product and a bivector part given by

$$
\underline{x} \wedge \underline{y}=\sum_{i<j} e_{i} e_{j}\left(x_{i} y_{j}-x_{j} y_{i}\right)=\frac{1}{2}(\underline{x} \underline{y}-\underline{y} \underline{x})
$$

being called the wedge product and corresponding to the outer product in $\mathbb{R}^{m}$.

Example 3. Let $m=3$. The 8-dimensional Clifford algebra $\mathbb{R}_{0,3}$ has the basis

$$
\left(1, e_{1}, e_{2}, e_{3}, e_{12}, e_{13}, e_{23}, e_{123}\right)
$$

consisting of the scalar 1 , the vectors $e_{1}, e_{2}, e_{3}$, the bivectors $e_{12}=e_{1} e_{2}, e_{13}=e_{1} e_{3}, e_{12}=e_{1} e_{2}$ and the trivector or pseudoscalar $e_{123}=e_{1} e_{2} e_{3}$ for which $e_{123}^{2}=1$. Note that the cross product of two vectors in $\mathbb{R}^{3}$ may be expressed as

$$
\underline{x} \times \underline{y}=-\underline{x} \wedge \underline{y} e_{123}
$$

Exploring further the multivector structure of $\mathbb{R}_{0, m}$ one may also write

$$
\mathbb{R}_{0, m}=\sum_{k \text { even }} \oplus \mathbb{R}_{0, m}^{k} \bigoplus \sum_{k \text { odd }} \oplus \mathbb{R}_{0, m}^{k}
$$

splitting the Clifford algebra into its even subalgebra

$$
\mathbb{R}_{0, m}^{+}=\sum_{k \text { even }} \oplus \mathbb{R}_{0, m}^{k}=\mathbb{R}_{0, m}^{0} \oplus \mathbb{R}_{0, m}^{2} \oplus \mathbb{R}_{0, m}^{4} \oplus \ldots
$$

and its odd subspace

$$
\mathbb{R}_{0, m}^{-}=\sum_{k \text { odd }} \oplus \mathbb{R}_{0, m}^{k}=\mathbb{R}_{0, m}^{1} \oplus \mathbb{R}_{0, m}^{3} \oplus \mathbb{R}_{0, m}^{5} \oplus \ldots
$$

The even subalgebra of $\mathbb{R}_{0, m}$ is isomorphic with $\mathbb{R}_{0, m-1}$, since it is generated by the elements $\varepsilon_{i}=e_{i} e_{m}, i=1, \ldots, m-1$, which satisfy the multiplication rules $\varepsilon_{i} \varepsilon_{j}+\varepsilon_{j} \varepsilon_{i}=-2 \delta_{i j}$ and can be interpreted as an orthonormal basis for $\mathbb{R}^{0, m-1}$.

The center of the Clifford algebra is given by $\mathbb{R}$ when $m$ is even and by $\mathbb{R} \oplus \mathbb{R} e_{M}$ when $m$ is odd.

In general the Clifford algebra will also possess zero divisors.

Example 4. Let again $m=2$. It is readily verified that the center of $\mathbb{R}_{0,2}$ only consists of the scalars, while its even subalgebra $\mathbb{R}_{0,2}^{+}$may be identified with $\mathbb{C}$, since it is generated by one single element $\varepsilon_{1}=e_{1} e_{2}$ for which it holds that $\varepsilon_{1}^{2}=-1$

Example 5. Now reconsider $m=3$. The center of $\mathbb{R}_{0,3}$ is given by $\mathbb{R} \oplus \mathbb{R} e_{123}$. For its even subalgebra it holds that $\mathbb{R} 0,3^{+} \simeq \mathbb{R}_{0,2} \simeq \mathbb{H}$, its generators being given by $\varepsilon_{1}=e_{1} e_{3}$ and $\varepsilon_{2}=e_{2} e_{3}$. By means of the central element $e_{123}$ it is easy to construct the zero divisors

$$
\varepsilon_{ \pm}=\frac{1}{2}\left(1 \pm e_{123}\right)
$$

submitting to the relations $\varepsilon_{ \pm}^{2}=\varepsilon_{ \pm}, \varepsilon_{+} \varepsilon_{-}=0=\varepsilon_{-} \varepsilon_{+}, \varepsilon_{+}+\varepsilon_{-}=1$. They induce a direct sum decomposition of the Clifford algebra $\mathbb{R}_{0,3}$ as follows:

$$
\mathbb{R}_{0,3} \simeq \varepsilon_{+} \mathbb{R}_{0,3}^{+} \oplus \varepsilon_{-} \mathbb{R}_{0,3}^{+} \simeq \mathbb{H} \oplus \mathbb{H}
$$


The above example may be generalized in the sense that, whenever $m=4 \ell+3$, it holds that the central element $e_{M}$ squares to +1 , whence the corresponding Clifford algebra splits as

$$
\mathbb{R}_{0,4 \ell+3}=\varepsilon_{+} \mathbb{R}_{0,4 \ell+3}^{+} \oplus \varepsilon_{-} \mathbb{R}_{0,4 \ell+3}^{+} \simeq \mathbb{R}_{0,4 \ell+2} \oplus \mathbb{R}_{0,4 \ell+2}
$$

by means of the zero divisors $\varepsilon_{ \pm}=\frac{1}{2}\left(1 \pm e_{M}\right)$, acting as projection operators. On the other hand, whenever $m=4 \ell+1$, the central element $e_{M}$ squares to -1 , whence it can be identified with the imaginary unit $i$ and one has

$$
\mathbb{R}_{0,4 \ell+1} \simeq \mathbb{C} \otimes \mathbb{R}_{0,4 \ell+1}^{+} \simeq \mathbb{C} \times \mathbb{R}_{0,4 \ell}
$$

\subsection{Complex Clifford algebras}

The complex Clifford algebra $\mathbb{C}_{m+1}$ can be introduced as

$$
\mathbb{C}_{m}=\mathbb{C} \otimes \mathbb{R}_{0, m}
$$

meaning that one takes the same generators $\left(e_{1}, \ldots, e_{m}\right)$ as above, with the same multiplication rules, however allowing for complex constants. As a linear associative algebra over $\mathbb{C}, \mathbb{C}_{m}$ has dimension $2^{m}$, since the set (1) still constitutes a basis. Any Clifford number $\lambda \in \mathbb{C}_{m}$ may thus be written as

$$
\lambda=\sum_{A} \lambda_{A} e_{A}, \quad \lambda_{A} \in \mathbb{C}
$$

or still as

$$
\lambda=a+i b, \quad a, b \in \mathbb{R}_{0, m}
$$

As above, consider the case where $m$ is odd, say $m=2 n+1$. The pseudoscalar $e_{M}=e_{1} \ldots e_{2 n+1}$ then is a central element of $\mathbb{C}_{2 n+1}$, with $e_{M}^{2}= \pm 1$. One thus may introduce a central element $\omega$ whose square is +1 in all cases, by putting

$$
\begin{array}{ll}
\omega=i e_{M}, & \text { when } e_{M}^{2}=-1 \text { i.e. when } n=2 \ell \\
\omega=e_{M}, & \text { when } e_{M}^{2}=+1 \text { i.e. when } n=2 \ell+1
\end{array}
$$

Then, by means of the projection operators $\varepsilon_{ \pm}=\frac{1}{2}(1 \pm \omega)$, a direct sum decomposition of $\mathbb{C}_{2 n+1}$ is obtained:

$$
\mathbb{C}_{2 n+1}=\varepsilon_{+} \mathbb{C}_{2 n+1}^{+} \oplus \varepsilon_{-} \mathbb{C}_{2 n+1}^{+} \simeq \mathbb{C}_{2 n} \oplus \mathbb{C}_{2 n}
$$

since the property is inherited from the real case that the even subalgebra $\mathbb{C}_{2 n+1}^{+}$of the Clifford algebra $\mathbb{C}_{2 n+1}$ is isomorphic to the Clifford algebra $\mathbb{C}_{2 n}$.

The whole multivector structure observed in $\mathbb{R}_{0, m}$ obviously is transferred to $\mathbb{C}_{m}$ as well.

\subsection{Involutions, inner product and norm}

Three (anti-)involutions are defined on the real Clifford algebra $\mathbb{R}_{0, m}$. They are introduced first on the basic elements $e_{A}$ and extended by linearity to the whole of the Clifford algebra.

(i) The main involution $a \mapsto \widetilde{a}$

$$
\begin{aligned}
\widetilde{e_{j}} & =-e_{j}, & & j=1, \ldots, m \\
\widetilde{a b} & =\widetilde{a} \widetilde{b}, & & a, b \in \mathbb{R}_{0, m}
\end{aligned}
$$


It directly follows that $\widetilde{e_{A}}=(-1)^{k} e_{A}$, whenever $|A|=k$, whence splitting an arbitrary Clifford number $a$ in its even and odd parts as $a=[a]_{+}+[a]_{-}$, one has

$$
\widetilde{a}=[a]_{+}-[a]_{-}=[a]_{0}-[a]_{1}+[a]_{2}-[a]_{3}+\ldots
$$

In particular $\widetilde{a}=a$, for $a \in \mathbb{R}_{0, m}^{+}$.

(ii) The reversion $a \mapsto a^{*}$

$$
\begin{array}{rlr}
e_{j}^{*} & =e_{j}, \quad j=1, \ldots, m \\
(a b)^{*} & =b^{*} a^{*}, \quad a, b \in \mathbb{R}_{0, m}
\end{array}
$$

Here one has $e_{A}^{*}=e_{\alpha_{k}} \ldots e_{\alpha_{1}}$ when $e_{A}=e_{\alpha_{1}} \ldots e_{\alpha_{k}}$, or still $e_{A}^{*}=(-1)^{\frac{k(k-1)}{2}} e_{A}$ whenever $|A|=k$. Thence

$$
a^{*}=[a]_{0}+[a]_{1}-[a]_{2}-[a]_{3}+[a]_{4}+[a]_{5}-\ldots
$$

(iii) The conjugation $a \mapsto \bar{a}$

$$
\begin{aligned}
\overline{e_{j}} & =-e_{j}, & & j=1, \ldots, m \\
\overline{a b} & =\bar{b} \bar{a}, & & a, b \in \mathbb{R}_{0, m}
\end{aligned}
$$

It clearly holds that the conjugation is the composition of the main involution and the reversion, whence $\overline{e_{A}}=(-1)^{\frac{k(k+1)}{2}} e_{A}$ whenever $|A|=k$. For an arbitrary Clifford number, one now has

$$
\bar{a}=[a]_{0}-[a]_{1}-[a]_{2}+[a]_{3}+[a]_{4}-[a]_{5}-\ldots
$$

The (anti-)involutions introduced on $\mathbb{R}_{0, m}$ clearly may be extended to $\mathbb{C}_{m}$, while keeping the same notations. However, an additional anti-involution on $\mathbb{C}_{m}$ is obtained by taking the tensor product of the conjugation on $\mathbb{R}_{0, m}$ and the classical complex conjugation on $\mathbb{C}$; this anti-involution is called the Hermitian conjugation and denoted by ${ }^{\dagger}$; explicitly, one has for an arbitrary Clifford number $\lambda \in \mathbb{C}_{m}$ :

$$
\lambda^{\dagger}=\bar{a}-i \bar{b}
$$

when starting from the expression (2), or

$$
\lambda^{\dagger}=\sum_{A} \lambda_{A}^{c} \overline{e_{A}}
$$

when starting from (3) and introducing the notation ${ }^{c}$ for the complex conjugation.

By means of the conjugation one may introduce an inner product and associated norm on $\mathbb{R}_{0, m}$, respectively given by

$$
\langle a, b\rangle=[\bar{a} b]_{0}=[b \bar{a}]_{0}
$$

and

$$
|a|=\sqrt{[a \bar{a}]_{0}}=\sqrt{\sum_{A} a_{A}^{2}}
$$

Similarly, one introduces an inner product and associated norm on $\mathbb{C}_{m}$ by means of the Hermitian conjugation:

$$
(\lambda, \mu)=\left[\lambda^{\dagger} \mu\right]_{0}=\left[\mu \lambda^{\dagger}\right]_{0} ; \quad|\lambda|=\sqrt{\left[\lambda \lambda^{\dagger}\right]_{0}}=\sqrt{\left.\sum_{A} \lambda_{A}\right|^{2}}
$$




\subsection{Geometric aspects and the spin group}

Recall the fact that the Clifford product of two vectors $\underline{v}=\sum_{j=0}^{m} v_{j} e_{j}$ and $\underline{w}=\sum_{j=0}^{m} w_{j} e_{j}$ decomposes as

$$
\underline{v} \underline{w}=[\underline{v} \underline{w}]_{0}+[\underline{v} \underline{w}]_{2}=\underline{v} \bullet \underline{w}+\underline{v} \wedge \underline{w}
$$

where

$$
[\underline{v} \underline{w}]_{0}=\underline{v} \bullet \underline{w}=\frac{1}{2}(\underline{v} \underline{w}+\underline{w} \underline{v})=-|\underline{v}||\underline{w}| \cos (\theta)
$$

and

$$
[\underline{v} \underline{w}]_{2}=\underline{v} \wedge \underline{w}=\frac{1}{2}(\underline{v} \underline{w}-\underline{w} \underline{v})=|\underline{v}||\underline{w}| \sin (\theta) \varepsilon_{1} \wedge \varepsilon_{2}
$$

with $\theta$ the angle between both vectors and $\left(\varepsilon_{1}, \varepsilon_{2}\right)$ an orthonormal basis (or frame) in the plane spanned by both vectors, with $\varepsilon_{1} \| \underline{v}$. When $\underline{v}$ and $\underline{w}$ are linearly dependent, one puts $\underline{v} \wedge \underline{w}=0$.

Inspired by this, a similar observation can be made for the case of the product of a vector $\underline{v}$ with a $k$-vector $a$. This product decomposes as

$$
\underline{v} a=[\underline{v} a]_{k-1}+[\underline{v} a]_{k+1} \equiv \underline{v} \bullet a+\underline{v} \wedge a
$$

where

$$
\begin{aligned}
\underline{v} \bullet a & =[\underline{v} a]_{k-1}=\frac{1}{2}\left(\underline{v} a-(-1)^{k} a \underline{v}\right) \\
\underline{v} \wedge a & =[\underline{v} a]_{k+1}=\frac{1}{2}\left(\underline{v} a+(-1)^{k} a \underline{v}\right)
\end{aligned}
$$

Now let $\underline{\omega}$ be a vector in $\mathbb{R}^{m}$ with norm $|\underline{\omega}|=1$, i.e. $\underline{\omega}$ is an element of the unit sphere $S^{m-1} \subset \mathbb{R}^{m}$. Any vector $\underline{x}$ in $\mathbb{R}^{m}$ may then be decomposed as a sum of two terms, one being parallel with $\underline{\omega}$ and the other being perpendicular to $\underline{\omega}$ :

$$
\underline{x}=\underline{x}_{\| \underline{\omega}}+\underline{x}_{\perp \underline{\omega}}=\langle\underline{x}, \underline{\omega}\rangle \underline{\omega}+\underline{\omega}(\underline{x} \wedge \underline{\omega})
$$

It is easily verified that the reflection of $\underline{x}$ with respect to the hyperplane perpendicular to $\underline{\omega}$ is given by

$$
R_{\underline{\omega}}(\underline{x})=-\underline{x}_{\| \underline{\omega}}+\underline{x}_{\perp \underline{\omega}}=-\langle\underline{x}, \underline{\omega}\rangle \underline{\omega}+\underline{\omega}(\underline{x} \wedge \underline{\omega})=\underline{\omega} \underline{x} \underline{\omega}
$$

Seen the fact that every rotation can be written as the composition of an even number of reflections, there should, for a given $T \in \mathrm{SO}(m)$, exist vectors $\underline{\omega}_{1}, \ldots, \underline{\omega}_{2 \ell}$ in $S^{m}$ such that $T=R_{\underline{\omega}_{1}} \ldots R_{\underline{\omega}_{2 \ell}}$. Hence the image of a vector $\underline{x}$ under the action of $T$ can be written as

$$
T[\underline{x}]=\underline{\omega}_{1} \underline{\omega}_{2} \ldots \underline{\omega}_{2 \ell} \underline{x}_{2 \ell} \underline{\omega}_{2 \ell} \underline{\omega}_{2} \underline{\omega}_{1}=s \underline{x} \bar{s}
$$

where $s=\underline{\omega}_{1} \underline{\omega}_{2} \ldots \underline{\omega}_{2 \ell}$ is a so-called spin element, belonging to the spin group of the Clifford algebra:

$$
\operatorname{Spin}(m)=\left\{s \in \mathbb{R}_{0, m} \mid s=\underline{\omega}_{1} \underline{\omega}_{2} \ldots \underline{\omega}_{2 \ell}, \underline{\omega}_{j}^{2}=-1, j=1, \ldots, 2 \ell\right\}
$$

With every spin element $s$ thus corresponds an action $h(s) \in S O(m)$ on the vectors, given by $h(s)[\underline{x}]=s \underline{x} \bar{s}$, and conversely, the spin group constitutes a double covering of $S O(m)$. The action of a spin element $s$ can be extended to the whole of the Clifford algebra, i.e.

$$
h(s)[a]=s a \bar{s}, \quad a \in \mathbb{R}_{0, m}
$$

and respects the multivector structure. On a Clifford algebra valued function $f(\underline{x})$, a spin element $s$ thus will act as $\bar{s} f(s \underline{x} \bar{s}) s$. 
Example 6. Consider two orthogonal vectors $\underline{\varepsilon}_{1}, \underline{\varepsilon}_{2}$ belonging to the unit sphere. Putting

$$
s=\exp \left(\theta \frac{\varepsilon_{1} \underline{\varepsilon}_{2}}{2}\right)
$$

it holds that $s$ is a spin element, which acts as follows:

$$
\begin{aligned}
& s \underline{\varepsilon}_{1} \bar{s}=\cos (\theta) \underline{\varepsilon}_{1}+\sin (\theta) \underline{\varepsilon}_{2} \\
& s \underline{\varepsilon}_{2} \bar{s}=-\sin (\theta) \underline{\varepsilon}_{1}+\cos (\theta) \underline{\varepsilon}_{2}
\end{aligned}
$$

and $s \underline{v} \bar{s}=\underline{v}, \forall \underline{v} \perp\left(\underline{\varepsilon}_{1}, \underline{\varepsilon}_{2}\right)$. The element $s$ thus represents the rotation over $\theta \in \mathbb{R}$ in the plane with orthonormal basis $\left(\underline{\varepsilon}_{1}, \underline{\varepsilon}_{2}\right)$.

The above example is important, since it was proven by Hamilton that every rotation $T$ can be rewritten as a composition of commuting plane rotations. Identifying $T$ with the action $h(s)$ of the spin element $s$, every spin element may thus be written as

$$
s=\exp \left(\theta_{1} \frac{\underline{\varepsilon}_{1} \underline{\varepsilon}_{2}}{2}\right) \exp \left(\theta_{2} \frac{\underline{\varepsilon}_{3} \underline{\varepsilon}_{4}}{2}\right) \ldots \exp \left(\theta_{\ell} \frac{\underline{\varepsilon}_{2 \ell-1} \underline{\varepsilon}_{2 \ell}}{2}\right)=\exp \left(\frac{1}{2}\left(\theta_{1} \underline{\varepsilon}_{1} \underline{\varepsilon}_{2}+\theta_{2} \underline{\varepsilon}_{3} \underline{\varepsilon}_{4}+\ldots+\theta_{\ell} \underline{\varepsilon}_{2 \ell-1} \underline{\varepsilon}_{2 \ell}\right)\right.
$$

where $\left(\underline{\varepsilon}_{1}, \ldots, \underline{\varepsilon}_{2 \ell}\right)$ is a frame and moreover $\theta_{1} \geq \theta_{2} \geq \ldots \geq \theta_{\ell}>0$. Summarizing, a spin element takes the form

$$
s=\exp (b), \text { with } b=\sum_{i<j} b_{i j} e_{i j} \in \mathbb{R}_{0, m}^{2}
$$

The interested reader may be referred to the book by Delanghe, Sommen and Souček for a thorough treatment of these group actions and their interpretation ([Delanghe et al, 1992]).

\section{Monogenic functions}

\subsection{Generalized Cauchy-Riemann operators}

The aim of this section is to introduce first order differential operators in $\mathbb{R}^{m+1}$ which linearize $\Delta_{m+1}$, the Laplace operator in $\mathbb{R}^{m+1}$. The considered functions are defined on $\mathbb{R}^{m+1}$ and take values in (subspace of) the real Clifford algebra $\mathbb{R}_{0, m}$ or its complexification $\mathbb{C}_{m}$. To fix the ideas, some examples in low dimension are considered.

Example 7. Let $m=1$. Consider the action of the operator $\partial_{x_{0}}+e_{1} \partial_{x_{1}}$ on functions $f: \mathbb{R}^{2} \rightarrow$ $\mathbb{R}_{0,1}$, i.e. consider the system

$$
\left(\partial_{x_{0}}+e_{1} \partial_{x_{1}}\right) f=0
$$

Identifying $x_{0}$ with $x, x_{1}$ with $y$ and the basis vector $e_{1}$ with the imaginary unit $i$, one obtains the Cauchy-Riemann equation in the complex plane, viz $\left(\partial_{x}+i \partial_{y}\right) f=0$, underlying the concept of holomorphy. Splitting the function $f$ into its real and imaginary parts as follows: $f=u+i v$, the equivalent system

$$
\partial_{x} u=\partial_{y} v, \quad \partial_{x} v=-\partial_{y} u
$$

is obtained. As is well-known, the standard Cauchy-Riemann operator indeed factorizes the 2dimensional Laplacian when being multiplied with its complex conjugate.

Example 8. Now let $m=3$ and consider functions $f: \mathbb{R}^{4} \rightarrow \mathbb{R}_{0,2}$, submitted to the action of the so-called Hamilton-Fueter operator:

$$
\left(\partial_{x_{0}}+e_{1} \partial_{x_{1}}+e_{2} \partial_{x_{2}}+e_{12} \partial_{x_{3}}\right) f=0
$$


Identification of the basis vectors $\left(e_{1}, e_{2}, e_{12}\right)$ with the quaternionic frame $(i, j, k)$ yields the equivalent form

$$
\left(\partial_{x_{0}}+i \partial_{x_{1}}+j \partial_{x_{2}}+k \partial_{x_{3}}\right) f=0
$$

When being multiplied with its quaternionic conjugate, this operator is seen to factorize the 4dimensional Laplacian.

In general, one considers functions $f\left(x_{0}, \ldots, x_{m}\right)$, i.e.

$$
f: \mathbb{R} \oplus \mathbb{R}^{m} \simeq \mathbb{R}^{m+1} \rightarrow \mathbb{R}_{m}\left(\text { or } \mathbb{C}_{m}\right)
$$

and the generalized Cauchy-Riemann operator

$$
D_{x}=\partial_{x_{0}}+\sum_{j=1}^{m} e_{j} \partial_{x_{j}}=\partial_{x_{0}}+\partial_{\underline{x}}
$$

also known as the Fueter-Delanghe operator, since it was first introduced in the works of R. Fueter ([Fueter, 1935a]) and, in full generality, of R. Delanghe ([Delanghe,1970]). The function $f$ is called left-monogenic in a domain $\Omega \subset \mathbb{R}^{m+1}$ if and only if it is in $\Omega$ a null solution of the above CauchyRiemann operator, i.e. if

$$
D_{x} f \equiv\left(\partial_{x_{0}}+\sum_{j=1}^{m} e_{j} \partial_{x_{j}}\right) f=0, \quad \forall\left(x_{0}, \ldots, x_{m}\right) \in \Omega
$$

Since multiplication in the Clifford algebra is non commutative, also the notion of right-monogenicity can be introduced. However, in this chapter, the focus will be on left-monogenic functions.

As indicated above, the generalized Cauchy-Riemann operator $D_{x}$ splits into a scalar part $\partial_{x_{0}}$ and an $m$-dimensional vectorial part

$$
\partial_{\underline{x}}=\sum_{j=1}^{m} e_{j} \partial_{x_{j}}
$$

called the Dirac operator. This operator is the Fourier or Fischer dual of the vector variable $\underline{x}=\sum_{j=1} e_{j} x_{j}$, and plays an important role as well, since it factorizes the $m$-dimensional Laplacian:

$$
\partial_{\underline{x}}^{2}=-\sum_{j=1}^{m} \partial_{x_{j}}^{2}=-\Delta_{m}
$$

while the Cauchy-Riemann operator factorizes the $m+1$-dimensional Laplacian:

$$
D_{x} \bar{D}_{x}=\left(\partial_{x_{0}}+\partial_{\underline{x}}\right)\left(\partial_{x_{0}}-\partial_{\underline{x}}\right)=\sum_{j=0}^{m} \partial_{x_{j}}^{2}=\Delta_{m+1}
$$

An alternative notion of monogenicity could thus be (and has been) associated to the null solution of this Dirac operator. There can however be shown to exist an equivalence between both notions, as will be explained in what follows.

To this end one needs to add a generator $e_{0}$ to the framework and consider the corresponding Clifford algebra $\mathbb{R}_{0, m+1}$. Then there are two ways of identifying $\mathbb{R}^{m+1}$ with a subspace of $\mathbb{R}_{0, m+1}$. First, let $x=\left(x_{0}, \underline{x}\right)=\left(x_{0}, x_{1}, \ldots, x_{m}\right)$ correspond to

$$
x=\sum_{j=0}^{m} x_{j} e_{j}=x_{0} e_{0}+\underline{x}
$$


i.e. $x \in \mathbb{R}_{0, m+1}^{1}$. Alternatively, put

$$
x=x_{0}+\sum_{j=1}^{m} x_{j} \overline{e_{0}} e_{j}=x_{0}+\sum_{j=1}^{m} x_{j} \varepsilon_{j}
$$

i.e. $x$ is paravector (a sum of a scalar and a vector) in $\mathbb{R}_{0, m} \simeq \mathbb{R}_{0, m+1}^{+}$. Now, according to the first approach, introduce the Dirac operator $\partial_{x}$ in $m+1$ dimensions, i.e.

$$
\partial_{x}=\sum_{j=0}^{m} e_{j} \partial_{x_{j}}=e_{0} \partial_{x_{0}}+\partial_{\underline{x}}
$$

Multiplying this operator from the left by the vector $\overline{e_{0}}$ one has

$$
\overline{e_{0}} \partial_{x}=\sum_{j=0}^{m} \overline{e_{0}} e_{j} \partial_{x_{j}}=\partial_{x_{0}}+\overline{e_{0}} \partial_{\underline{x}}=\partial_{x_{0}}+\sum_{j=1}^{m} \overline{e_{0}} e_{j} \partial_{x_{j}}=\partial_{x_{0}}+\sum_{j=1}^{m} \varepsilon_{j} \partial_{x_{j}}
$$

which can be interpreted as a Cauchy-Riemann operator corresponding to the paravector form of $x$.

Both the Dirac operator and the Cauchy-Riemann operator are so-called rotation invariant operators. In the present framework this should be interpreted in the sense that both operators commute with the action of spin elements on functions, or still: the action of a spin element on a monogenic function will preserve its monogenicity.

In this respect it is interesting to mention the existence of so-called quaternionic analysis, which is not the same as Clifford analysis for quaternion-valued functions; in the paper by Bory Reyes and Shapiro, [Bory Reyes and Shapiro, 2010], the relation is explained between quaternionic analysis, where the so-called Fueter and the Moisil-Teodorescu operator are considered, and Clifford analysis for the Cauchy-Riemann and the Dirac operator.

Returning to $\mathbb{R}_{0, m}$, it also is an interesting topic to study solutions of either the Cauchy-Riemann or the Dirac equation with restricted values, i.e. functions taking values in only one of the components of the multivector structure of the Clifford algebra. The resulting systems have received many attention in the literature.

Example 9. Let $f$ be a vector valued function, i.e. $f=\sum_{j=1}^{m} f_{j} e_{j}$. Expressing the fact that $f$ should be a null solution of the m-dimensional Dirac operator results in the splitting of the corresponding equation into the following system, called Riesz system:

$$
\partial_{\underline{x}} \bullet f=0, \quad \partial_{\underline{x}} \wedge f=0
$$

where

$$
\begin{aligned}
\partial_{\underline{x}} \bullet f & =-\sum_{j=1}^{m} \partial_{x_{j}} f_{j}=-\operatorname{div} f \\
\partial_{\underline{x}} \wedge f & =\sum_{i<j} e_{i j}\left(\partial_{x_{i}} f_{j}-\partial_{x_{j}} f_{i}\right)=\operatorname{rot} f
\end{aligned}
$$

A vector valued monogenic function thus is a rotation and divergence free vector field, as considered by Stein ([Stein,1970]).

Example 10. The generalization of the above example is the so-called Hodge system, obtained by restricting the values of $f$ to an arbitrary but fixed space of $k$-vectors $\mathbb{R}_{0, m}^{k}$. The equation 
then splits into a system which formally can be written again as (4), but takes another explicit form, respectively in $\mathbb{R}_{0, m}^{(k-1)}$ and $\mathbb{R}_{0, m}^{(k+1)}$. Note that in such a case, left and right monogenicity are equivalent, since

$$
\partial_{\underline{x}} f=0 \Rightarrow \partial_{\underline{x}} \bullet f=0 \text { and } \partial_{\underline{x}} \wedge f=0 \Rightarrow f \partial_{\underline{x}}
$$

Moreover, conversely it holds that if a function is both left and right monogenic, then each of its $k$-vector parts is monogenic.

Since products of monogenic functions are, in general, no longer monogenic, it is particularly interesting to develop alternative methods for constructing examples, or even classes, of monogenic functions. A number of these methods and approaches are briefly discussed in the remainder of this section.

\subsection{The Cauchy-Kovalevskaya extension}

The Cauchy-Kovalevskaya extension theorem ([Cauchy,1882, Kowalevsky,1875]) has a long history; for a detailed account the reader is referred to [Cooke]. In its most simple setting, it reads as follows.

Theorem 1. If the functions $F, f_{0}, \ldots, f_{k-1}$ are analytic in a neighbourhood of the origin, then the initial value problem

$$
\begin{aligned}
\partial_{t}^{k} h(\underline{x}, t) & =F\left(\underline{x}, t, \partial_{t}^{i} \partial_{\underline{x}}^{\alpha} h\right) \\
\partial_{t}^{j} h(\underline{x}, 0) & =f_{j}(\underline{x}), \quad j=0, \ldots, k-1
\end{aligned}
$$

has a unique solution which is analytic in a neighbourhood of the origin, provided that $|\alpha|+i \leq k$.

In the case where the differential operator involved is the classical Cauchy-Riemann operator in the complex plane, i.e. when the differential equation reduces to $\partial_{t} h=-i \partial_{x} h$ (with $k=1,|\alpha|=1, i=$ 0 ), the theorem states that a holomorphic function in an appropriate region of the complex plane is completely determined by its restriction to the real axis. In that case, reverting to the traditional notations in the complex plane, one has the following construction formula for the holomorphic Cauchy-Kovalevskaya (short: CK) extension.

Proposition 1. If the function $f_{0}(x)$ is real-analytic in $|x|<a$, then

$$
F(z)=\exp \left(i y \frac{d}{d x}\right)\left[f_{0}(x)\right]=\sum_{k=0}^{\infty} \frac{1}{k !} i^{k} y^{k} f_{0}^{(k)}(x)
$$

is holomorphic in $|z|<a$ and $\left.F(z)\right|_{\mathbb{R}}=f_{0}(x)$.

The CK extension theorem has a direct generalization in Clifford analysis; it reads as follows.

Theorem 2. If $f\left(x_{1}, x_{2}, \ldots, x_{m}\right)$ is real-analytic in an open set $\Omega$ of $\mathbb{R}^{m}$ identified with $\{x \in$ $\left.\mathbb{R}^{m+1}: x_{0}=0\right\}$, then there exists an open neighbourhood $\widetilde{\Omega}$ of $\Omega$ in $\mathbb{R}^{m+1}$ and a unique monogenic function $F$ (w.r.t. $D_{x}$ ) in $\widetilde{\Omega}$ such that its restriction to $\Omega$ precisely is $f$. If moreover $\Omega$ contains the origin, then in an open neigbourhood of the origin this CK-extension $F$ is given by

$$
F\left(x_{0}, x_{1}, \ldots, x_{m}\right)=\exp \left(-x_{0} \partial_{\underline{x}}\right) f(\underline{x})=\sum_{k=0}^{\infty} \frac{1}{k !}\left(-x_{0}\right)^{k} \partial_{\underline{x}}^{k} f(\underline{x})
$$

This theorem is very powerful, as it allows to generate monogenic functions starting from realanalytic ones. An important example concerns the CK-entension of the Gaussian, leading to the introduction of the Clifford-Hermite polynomials. 
Example 11. A direct calculation yields

$$
\mathrm{CK}\left(\exp \left(-\frac{|\underline{x}|^{2}}{2}\right)\right)=\sum_{k=0}^{+\infty} \frac{\left(-x_{0}\right)^{k}}{k !} H_{k}(\underline{x}) \exp \left(-\frac{|\underline{x}|^{2}}{2}\right)
$$

where

$$
H_{k}(\underline{x})=\exp \left(\frac{|\underline{x}|^{2}}{2}\right) \partial_{\underline{x}}^{k} \exp \left(-\frac{|\underline{x}|^{2}}{2}\right)
$$

is a polynomial of degree $k$, called Clifford-Hermite polynomial. Explicitly, the Clifford-Hermite polynomials are given by

$$
H_{k}(\underline{x})= \begin{cases}2^{\frac{k}{2}} \Gamma\left(\frac{k}{2}+1\right) L_{\frac{k}{2}}^{\frac{m}{2}-1}\left(\frac{|\underline{x}|^{2}}{2}\right), & k \text { even } \\ 2^{\frac{k-1}{2}} \Gamma\left(\frac{k+1}{2}\right) L_{\frac{k-1}{2}}^{\frac{m}{2}}\left(\frac{|\underline{x}|^{2}}{2}\right), & k \text { odd }\end{cases}
$$

in terms of the Laguerre polynomials on the real line. They form a direct generalization of the real Hermite polynomials, not only formally, but also for what concerns their properties and behaviour, and were used as kernel functions in a higher dimensional continuous wavelet transform, see e.g. [Brackx and Sommen, 2000].

Another important application of the CK extension theorem is the construction of so-called monogenic plane waves, of which the following function is an example.

Example 12. Let $f(\underline{x})=\exp (i\langle\underline{x}, \underline{t}\rangle)$, with $\underline{t} \in \mathbb{R}^{m}$ a parameter, then

$$
\mathrm{CK}(\exp (i\langle\underline{x}, \underline{t}\rangle))=\left(\cosh \left(x_{0}|\underline{t}|\right)+i \frac{\underline{t}}{|\underline{t}|} \sinh \left(x_{0}|\underline{t}|\right)\right) \exp (i\langle\underline{x}, \underline{t}\rangle)
$$

Note that in the complex plane case one has $\mathrm{CK}(\exp (i x t))=\exp (i z t)$

In general one starts from a function $f: \mathbb{R} \rightarrow \mathbb{R}($ or $\mathbb{C})$, and one considers $f(\langle\underline{x}, \underline{t}\rangle)$ where $\underline{t} \in \mathbb{R}^{m}$ is a parameter. The name plane wave originates from the fact that, for a given $\underline{t} \in \mathbb{R}^{m}, f(\langle\underline{x}, \underline{t}\rangle)$ is constant on planes perpendicular to $\underline{t}$. The CK-extension of such a function is given by

$$
\left.\mathrm{CK}(f(\langle\underline{x}, \underline{t}\rangle))=f\left(\langle\underline{x}, \underline{t}\rangle-x_{0} \underline{t}\right)=f_{1}\left(\langle\underline{x}, \underline{t}\rangle, x_{0}|\underline{t}|\right)-\frac{\underline{t}}{|\underline{t}|} f_{2}\left(\langle\underline{x}, \underline{t}\rangle, x_{0}|\underline{t}|\right)\right)
$$

where $f_{1}$ and $f_{2}$ are such that $f_{1}(x, y)+i f_{2}(x, y)=f(x+i y)$ is the holomorphic extension of $f(x)$ in the complex plane. Summarizing, the monogenic CK-extension of $f(\langle\underline{x}, \underline{t}\rangle)$ is obtained by first constructing the holomorphic CK-extension $f(x+i y)$ of $f(x)$ and subsequently substituting $x$ by $\langle\underline{x}, \underline{t}\rangle, y$ by $x_{0}|\underline{t}|$ and $i$ by $-\frac{\underline{t}}{|\underline{t}|}$. Note that this is an accordance with the above example.

Example 13. Starting from the homogeneous powers $f(x)=x^{k}$ one obtains the monogenic plane waves $\left(\langle\underline{x}, \underline{t}\rangle-x_{0} \underline{t}\right)^{k}, k \in \mathbb{N}$ or $\mathbb{Z}$.

Example 14. An important example, in view of applications, is the so-called Radon kernel. It is obtained by starting from the Cauchy kernel in the complex plane, viz $\frac{1}{2 \pi i} \frac{1}{z}$ and considering the corresponding monogenic plane wave

$$
\frac{1}{2 \pi} \frac{\underline{t}}{\langle\underline{x}, \underline{t}\rangle-\underline{t} x_{0}}=\frac{1}{2 \pi} \frac{\langle\underline{x}, \underline{t}\rangle \underline{t}-x_{0}|\underline{t}|^{2}}{\langle\underline{x}, \underline{t}\rangle^{2}+x_{0}^{2}|\underline{t}|^{2}}
$$

For a fixed $\underline{t}$ this function has singularities whenever $x_{0}=0$ and $\langle\underline{x}, \underline{t}\rangle=0$. 
Starting from a real analytic function $f(x)$ and its holomorphic extension $f(z)$, the above identifications allow for the construction of two more associated monogenic functions. To this end, consider in $\mathbb{R}^{m}$ the functions

$$
\theta_{ \pm}=\frac{1}{2}\left(1 \pm i \frac{\underline{t}}{|\underline{t}|}\right)
$$

which act as projection operators since $\theta_{+}+\theta_{-}=1, \theta_{ \pm}^{2}=\theta_{ \pm}, \theta_{+} \theta_{-}=0$. These functions are called the Heaviside projectors since they generalize the Heaviside functions $Y( \pm t)$ on the real axis. Since moreover it holds that $\underline{t} \theta_{ \pm}=-i \theta_{ \pm} \mid \underline{t}$, it can be readily verified that the functions

$$
f\left(\langle\underline{x}, \underline{t}\rangle+x_{0}|\underline{t}|\right) \theta_{ \pm}
$$

are monogenic. This is the way in which monogenic Fourier kernels were constructed by T. Qian and A. Mc Intosh ([Li et al, 1994]), see also [Sommen, 1988] and [Brackx et al, 2005].

$$
\exp (i z) \longrightarrow \frac{1}{2}\left(1 \pm i \frac{\underline{t}}{|\underline{t}|}\right) \exp \left(i\langle\underline{x}, \underline{t}\rangle \mp x_{0}|\underline{t}|\right)
$$

In a similar way, two alternative monogenic Radon kernels can be considered, viz:

$$
\frac{1}{2 \pi i} \frac{1}{z} \longrightarrow \frac{1}{2 \pi} \frac{1}{\langle\underline{x}, \underline{t}\rangle \pm i x_{0}|\underline{t}|} \frac{1}{2}\left(1 \pm i \frac{\underline{t}}{|\underline{t}|}\right)=\frac{1}{4 \pi} \frac{|\underline{t}|+i \underline{t}}{\langle\underline{x}, \underline{t}\rangle|\underline{t}| \pm i x_{0}|\underline{t}|^{2}}
$$

Remark 1. One may also consider plane wave solutions of $\partial_{\underline{x}}$. Here it suffices to consider two orthogonal real vectors $\underline{t}, \underline{s} \in \mathbb{R}^{m}$ of equal norm, and the corresponding complex vector $\underline{\tau}=\underline{t}+i \underline{s}$. Under the present conditions it holds that

$$
\underline{\tau}^{2}=(\underline{t}+i \underline{s})^{2}=|\underline{s}|^{2}-|\underline{t}|^{2}-2 i\langle\underline{t}, \underline{s}\rangle=0
$$

whence it can be easily verified that, starting from a real-analytic function $f(x)$, the corresponding plane wave $f(\langle\underline{x}, \underline{\tau}\rangle) \underline{\tau}$ is monogenic w.r.t. $\partial_{\underline{x}}$.

\subsection{Axially symmetric monogenics}

The above examples and considerations reveal a tight connection between holomorphic functions of one complex variable and monogenic functions in $\mathbb{R}^{m}$ or $\mathbb{R}^{m+1}$. Therefore it is important to notice that, in general, a holomorphic function $f(x+i y)$ will not give rise to a monogenic function $f\left(x_{0}+\underline{x}\right)$. There is however a method to construct monogenic functions from holomorphic ones, given by the Fueter-Sce theorem, see e.g. [Fueter,1935b], [Sce,1957].

Theorem 3. Let $m$ be odd and let $f(x+i y)$ be a holomorphic function in the complex plane. Then the function

$$
\left(\Delta_{m+1}\right)^{\frac{m-1}{2}} f\left(x_{0}+\underline{x}\right)
$$

is monogenic.

In the case where $m$ is even, the above result was extended by T. Qian ([Qian, 1997]) using Fourier multiplier theory.

A particular class of monogenic functions are those with axial symmetry. Introducing spherical co-ordinates, i.e. writing $\underline{x}=\rho \underline{\omega}$, with $\rho=|\underline{x}|$ and $\underline{\omega} \in S^{m-1}$, a function is called axial monogenic if and only if it is a null solution of the Cauchy-Riemann operator, viz

$$
\left(\partial_{x_{0}}+\partial_{\underline{x}}\right) f\left(x_{0}, \underline{x}\right)=0
$$


and it takes the form

$$
f\left(x_{0}, \underline{x}\right)=A\left(x_{0}, \rho\right)+\underline{\omega} B\left(x_{0}, \rho\right)
$$

where $A$ and $B$ are scalar valued functions, see e.g. [Common and Sommen, 1993]. The components $A$ and $B$ thus have to submit to the following system:

$$
\begin{aligned}
\partial_{x_{0}} A-\partial_{\rho} B & =\frac{m-1}{\rho} B \\
\partial_{x_{0}} B+\partial_{\rho} A & =0
\end{aligned}
$$

also called the Vekua system. Note that in particular, the above mentioned Fueter-Sce theorem yields functions of this form.

A first important example is the so-called Cauchy kernel, underlying the Cauchy integral representation formula, studied below.

Example 15. The function

$$
C\left(x_{0}, \underline{x}\right)=\frac{1}{a_{m+1}} \frac{x_{0}-\underline{x}}{\left|x_{0}+\underline{x}\right|^{m+1}}=\frac{1}{a_{m+1}} \frac{x_{0}-\rho \underline{\omega}}{\left(x_{0}^{2}+\rho^{2}\right)^{\frac{m+1}{2}}}
$$

where $a_{m+1}$ is the area of the unit sphere $S^{m}$ in $\mathbb{R}^{m+1}$, is the fundamental solution of the CauchyRiemann operator, i.e.

$$
\left(\partial_{x_{0}}+\partial_{\underline{x}}\right) C\left(x_{0}, \underline{x}\right)=\delta\left(x_{0}\right) \delta(\underline{x})
$$

It thus is axial monogenic in $\mathbb{R}^{m+1} \backslash\{0\}$. Note in particular that, for a given function $g\left(x_{0}, \underline{x}\right)$ with compact support, the function

$$
u\left(x_{0}, \underline{x}\right)=[C * g]\left(x_{0}, \underline{x}\right)=\int_{\mathbb{R}^{m+1}} C\left(x_{0}-y_{0}, \underline{x}-\underline{y}\right) g\left(y_{0}, \underline{y}\right) d V(y)
$$

will solve the problem $D_{x} u=g$. Here $d V(y)$ denotes the volume element in $\mathbb{R}^{m+1}$, see also below.

Another example concerns the Clifford-Bessel function.

Example 16. Putting $\mathcal{E}\left(x_{0}, \underline{x}\right)=\exp \left(x_{0}\right) \mathcal{E}(\underline{x})$, the Vekua system leads to the following solution:

$$
\mathcal{E}(\underline{x})=2^{\frac{m}{2}-1} \Gamma\left(\frac{m}{2}\right) \rho^{1-\frac{m}{2}}\left(J_{\frac{m}{2}-1}(\rho)+\underline{\omega} J_{\frac{m}{2}}(\rho)\right)
$$

in terms of the Bessel function of the first kind on the real line.

Finally, it is also possible to construct axial monogenics starting from plane wave solutions. To this end, take $\underline{t} \in S^{m-1}$ and consider a monogenic plane wave of the form

$$
G\left(x_{0}, \underline{x}, \underline{t}\right)=g_{1}\left(\langle\underline{x}, \underline{t}\rangle, x_{0}\right)-\underline{t} g_{2}\left(\langle\underline{x}, \underline{t}\rangle, x_{0}\right)
$$

then the function

$$
I\left(x_{0}, \underline{x}\right)=\int_{S^{m-1}} G\left(x_{0}, \underline{x}, \underline{t}\right) d \underline{t}
$$

is axial monogenic. Indeed, invoking the theorem of Funk-Hecke, it can be verified that $I$ is monogenic and takes the form $I=A\left(x_{0}, \rho\right)+\underline{\omega} B\left(x_{0}, \rho\right)$, with

$$
\begin{aligned}
& A\left(x_{0}, \rho\right)=a_{m} \int_{-1}^{1} g_{1}\left(\rho s, x_{0}\right)\left(1-s^{2}\right)^{\frac{m-3}{2}} d s \\
& B\left(x_{0}, \rho\right)=-a_{m} \int_{-1}^{1} g_{2}\left(\rho s, x_{0}\right) s\left(1-s^{2}\right)^{\frac{m-3}{2}} d s
\end{aligned}
$$


Example 17. Starting from $G\left(x_{0}, \underline{x}, \underline{t}\right)=\frac{1}{2}(1+i \underline{t}) \exp \left(i\langle\underline{x}, \underline{t}\rangle-x_{0}\right)$, one obtains

$$
I\left(x_{0}, \underline{x}\right)=\frac{1}{2} a_{m} \exp \left(-x_{0}\right)\left(I_{0}(\rho)+i \underline{\omega} I_{1}(\rho)\right)
$$

with

$$
I_{k}(\rho) \sim i^{k} \rho^{1-\frac{m}{2}} J_{k+\frac{m}{2}-1}(\rho), \quad k=0,1
$$

whence $I\left(x_{0}, \underline{x}\right)$ equals, up to a multiplicative constant, the Clifford-Bessel function $\mathcal{E}\left(-x_{0},-\underline{x}\right)$.

An important result in this respect is the so-called Radon decomposition of the Cauchy kernel, which then leads to the Clifford-Radon transform, as for instance derived in [Sommen, 1992a], see also below.

Theorem 4. If $m$ is even, one has

$$
\frac{1}{a_{m+1}} \frac{x_{0}-\underline{x}}{\left|x_{0}+\underline{x}\right|^{m+1}}= \pm(-1)^{\frac{m}{2}} \frac{(m-1) !}{2(2 \pi)^{m}} \int_{S^{m-1}}\left(\langle\underline{x}, \underline{t}\rangle-x_{0} \underline{t}\right)^{-m} d \underline{t}
$$

where the plus sign has to be taken whenever $x_{0}>0$ and the minus sign whenever $x_{0}<0$. If $m$ is odd, one has

$$
\frac{1}{a_{m+1}} \frac{x_{0}-\underline{x}}{\left|x_{0}+\underline{x}\right|^{m+1}}=(-1)^{\frac{m+1}{2}} \frac{(m-1) !}{2(2 \pi)^{m}} \int_{S^{m-1}}\left(\langle\underline{x}, \underline{t}\rangle-x_{0} \underline{t}\right)^{-m} \underline{t} d \underline{t}
$$

\section{Cauchy integral formulae}

Within the theory of holomorphic functions in the complex plane, Cauchy's integral representation formula constitutes an essential result. It expresses the fact that a holomorphic function defined on a disk $D$ is completely determined by its values on the boundary $\partial D$ of the disk:

$$
f(z)=\frac{1}{2 \pi i} \int_{\partial D} \frac{f(\xi)}{\xi-z} d \xi, \quad z \in \stackrel{\circ}{D}
$$

and it equally provides integral formulae for all derivatives of this holomorphic function. In Clifford analysis a generalization of this formula has been established, as is briefly summarized in what follows, see e.g. [Brackx et al, 1982].

The present framework is the one where a generator $e_{0}$ is added and the corresponding Clifford algebra $\mathbb{R}_{0, m+1}$ is considered. The operator under consideration is the Dirac operator $\partial_{x}$ in $m+1$ dimensions, although all results mentioned may easily be adapted to the case of the CauchyRiemann operator $D_{x}$.

Let $\Omega \subset \mathbb{R}^{m+1}$ be open, and let $C \subset \Omega$ be a compact orientable $(m+1)$-dimensional manifold with $C^{\infty}$ smooth boundary $\partial C$. The vector valued oriented surface element on $\partial C$ is defined by the differential form

$$
d \sigma_{x}=\sum_{j=0}^{m}(-1)^{j} e_{j} d \widehat{x}_{j}
$$

where $d \widehat{x}_{j} \equiv d x_{0} \wedge \ldots \wedge\left[d x_{j}\right] \wedge \ldots \wedge d x_{m}=d x_{0} \wedge \ldots \wedge d x_{j-1} \wedge d x_{j+1} \wedge \ldots \wedge d x_{m}, j=0, \ldots, m$, while the corresponding volume element reads $d V(x)=d x_{0} \wedge \ldots \wedge d x_{m}$. Observe that, unlike the situation in real analysis, the ordering of the variables plays a role, since a change of order would result into a change of sign. Denoting the outwardly pointing unit normal vector at $x \in \partial C$ by $n(x)$, one may also write

$$
d \sigma_{x}=n(x) d S(x)
$$


where $\mathrm{dS}(\mathrm{x})$ denotes the elementary surface measure.

A first fundamental result is the Clifford-Stokes theorem.

Theorem 5. Let $f, g \in C_{1}(\Omega)$. Then

$$
\int_{\partial C} g(x) d \sigma_{x} f(x)=\int_{C}\left[\left(g \partial_{x}\right) f+g\left(\partial_{x} f\right)\right] d V(x)
$$

As an immediate consequence one obtains the basic theorem of Cauchy.

Theorem 6. Let the function $f$ be (left-)monogenic w.r.t. the Dirac operator $\partial_{x}$, then

$$
\int_{\partial C} d \sigma_{x} f(x)=0=\int_{\partial C} n(x) f(x) d S(x)
$$

Again, as a consequence of the non commutative multiplication, the order of the factors in the integrand should, in both statements above as well as in what follows, carefully be respected.

Example 18. A particular example is the following: take $f \equiv 1$ and let $C$ be the unit ball in $\mathbb{R}^{m+1}$, i.e. $\partial C=S^{m}$, the unit sphere in $\mathbb{R}^{m+1}$. Then at each point $\omega \in S^{m}, n(\omega)=\omega$, whence

$$
\int_{S^{m}} \omega d S(\omega)=0
$$

In Example 15 the so-called Cauchy-kernel $C\left(x_{0}, \underline{x}\right)$ was introduced as a fundamental solution of the Cauchy-Riemann operator $D_{x}$. In a similar way, one can easily verify that the function

$$
E(x) \equiv E\left(x_{0}, \underline{x}\right)=\frac{1}{a_{m+1}} \frac{\bar{x}}{|x|^{m+1}}
$$

is a fundamental solution of the Dirac operator $\partial_{x}$. The function $E$ is vector valued, locally integrable, tends to zero at infinity, and indeed submits to $\partial_{x} E=E \partial_{x}=\delta(x)$, whence it is left and right monogenic in $\mathbb{R}^{m+1} \backslash\{0\}$. Using this function as a kernel function, i.e. taking $g(\cdot)=E(\cdot-x)$ in Theorem 5, the following Clifford-Borel-Pompeiu formula is obtained.

Theorem 7. Let $f \in C_{1}(\Omega)$. Then

$$
\int_{\partial C} E(y-x) d \sigma_{y} f(y)-\int_{C} E(y-x)\left(\partial_{y} f\right) d V(y)= \begin{cases}0 & \text { if } x \in \Omega \backslash C \\ f(x) & \text { if } x \in \stackrel{\circ}{C}\end{cases}
$$

The Clifford-Cauchy integral representation formula then is nothing but a corollary of the above statement for the case of monogenic functions.

Theorem 8. Let $f$ be (left-)monogenic in $\Omega$. Then

$$
\int_{\partial C} E(y-x) d \sigma_{y} f(y)= \begin{cases}0 & \text { if } x \in \Omega \backslash C \\ f(x) & \text { if } x \in \stackrel{\circ}{C}\end{cases}
$$

Remark 2. When $\Omega=\mathbb{R}^{m+1}$, one may, in all of the above, take $C=\mathbb{R}_{ \pm}^{m+1}$ (the upper or lower half space). Then in both cases $\partial C \simeq \mathbb{R}^{m}$, the latter being identified with the hyperplane $x_{0}=0$. 


\section{Applications: integral transforms}

\subsection{The Clifford-Cauchy and the Clifford-Hilbert transform}

The Cauchy transform is a well-known integral operator in the complex plane, which applies to functions defined on the boundary of a domain, and associates to them a function which is holomorphic both in the interior and exterior of that domain. In particular, let $f \in L_{2}(\partial D), D$ being the unit disk, then the Cauchy transform of $f$ is given by

$$
\mathcal{C}[f](z)=\frac{1}{2 \pi i} \int_{\partial D} \frac{f(\zeta)}{\zeta-z} d \zeta, \quad z \notin \partial D
$$

yielding a function which is holomorphic in $\mathbb{C} \backslash \partial D$ and tends to zero when $z \rightarrow \infty$.

Although this probably is the most well-known version, it is not the one which will be considered here. In view of possible applications, it is particularly interesting to consider $f \in L_{2}(\mathbb{R}), \mathbb{R}$ being considered as the boundary of both the upper and lower half plane. In that case

$$
\mathcal{C}[f](x+i y)=\mathcal{C}[f](z)=\frac{1}{2 \pi i} \int_{\mathbb{R}} \frac{f(t)}{t-z} d t=\frac{1}{2 \pi i} \int_{\mathbb{R}} \frac{f(t)}{(t-x)-i y} d t, \quad z \notin \mathbb{R}
$$

yields a function which is holomorphic in the upper and lower half plane and tends to zero when $y \rightarrow \infty$. Looking at the jump over the real axis made by the Cauchy kernel, one has

$$
\lim _{\varepsilon \rightarrow 0+} \frac{1}{2 \pi i}\left(\frac{1}{x+i \varepsilon}-\frac{1}{x-i \varepsilon}\right)=-\lim _{\varepsilon \rightarrow 0} \frac{1}{\pi} \frac{\varepsilon}{x^{2}+\varepsilon^{2}}=-\delta(x)
$$

and more in detail, looking at the upper and lower limit separately:

$$
\lim _{\varepsilon \rightarrow 0+} \frac{1}{2 \pi i} \frac{1}{x \pm i \varepsilon}=\mp \frac{1}{2}\left(\delta(x) \pm \frac{1}{\pi i} \operatorname{Pv} \frac{1}{x}\right)
$$

whence it holds that

$$
f(x)=\lim _{\varepsilon \rightarrow 0+}(\mathcal{C}[f](x+i \varepsilon)-\mathcal{C}[f](x-i \varepsilon))
$$

while

$$
H[f](x) \equiv \lim _{\varepsilon \rightarrow 0+}(\mathcal{C}[f](x+i \varepsilon)+\mathcal{C}[f](x-i \varepsilon))=\frac{1}{\pi i} \int_{\mathbb{R}} \frac{f(t)}{t-x} d t=\left[-\frac{1}{\pi i} \operatorname{Pv} \frac{1}{\cdot} * f(\cdot)\right](x)
$$

defines the so-called Hilbert transform on the real line, as the convolution with the Hilbert kernel. This Hilbert transform is an important tool in engineering sciences, and in particular in signal analysis, where filters are designed for obtaining information on various signal properties, based on the notion of analytic signal, which is a linear combination of a bandpass filter and its Hilbert transform, see [Hahn, 1996].

The above relations between the Cauchy transform, the Hilbert transform and the original function may also be rewritten in the following form:

$$
\lim _{y \rightarrow 0 \pm} \mathcal{C}[f](x+i y)= \pm \frac{1}{2} f(x)+\frac{1}{2} H[f](x)
$$

known as the Plemelj-Sokhotski formulae. The analytic signal then is the non-tangential boundary value of the holomorphic Cauchy integral in the upper half plane, i.e. $\frac{1}{2} f(x)+\frac{1}{2} H[f](x)$. 
When passing to higher dimension, the classical approach to introduce a Hilbert transform is a tensorial one, taking the so-called Riesz transforms in each of the variables. In the intrinsically multidimensional framework of Clifford analysis however, a truely higher dimensional Hilbert transform can be introduced. As above, the fundamental operator considered here is the Dirac operator $\partial_{x}$ in $m+1$ dimensions.

Let $f \in L_{2}\left(\mathbb{R}^{m}\right), \mathbb{R}^{m}$ being considered as the joint boundary of upper and lower half space in $\mathbb{R}^{m+1}$, identified as above with the hyperplane $x_{0}=0$. Then the Clifford-Cauchy transform of $f$ is given by

$$
\mathcal{C}[f](x) \equiv \mathcal{C}[f]\left(x_{0}, \underline{x}\right)=E\left(x_{0}, \cdot\right) * f(\cdot)(\underline{x})=-\frac{1}{a_{m+1}} \int_{\mathbb{R}^{m}} \frac{x_{0} e_{0}-(\underline{y}-\underline{x})}{\left|x_{0} e_{0}-(\underline{y}-\underline{x})\right|^{m+1}} f(\underline{y}) d \underline{y}
$$

where $E\left(x_{0}, \underline{x}\right)$ is the above introduced fundamental solution of the Dirac operator $\partial_{x}$. This yields a function which is monogenic w.r.t. $\partial_{x}$ in both half spaces and tends to zero at infinity.

Considering the jump of the fundamental solution over the boundary $\mathbb{R}^{m}$ one obtains

$$
\begin{aligned}
& \lim _{\varepsilon \rightarrow 0+} E(\varepsilon, \underline{x})-E(-\varepsilon, \underline{x})=\frac{2}{a_{m+1}} \overline{e_{0}} \lim _{\varepsilon \rightarrow 0} \frac{\varepsilon}{\left(|\underline{x}|^{2}+\varepsilon^{2}\right)^{\frac{m+1}{2}}}=\overline{e_{0}} \delta(\underline{x}) \\
& \lim _{\varepsilon \rightarrow 0+} E(\varepsilon, \underline{x})+E(-\varepsilon, \underline{x})=-\frac{2}{a_{m+1}} \lim _{\varepsilon \rightarrow 0} \frac{\underline{x}}{\left(|\underline{x}|^{2}+\varepsilon^{2}\right)^{\frac{m+1}{2}}}=-\frac{2}{a_{m+1}} \frac{\underline{x}}{|\underline{x}|^{m+1}}
\end{aligned}
$$

Thence, letting $x_{0}$ tend to zero yields following boundary limits:

$$
\lim _{x_{0} \rightarrow 0 \pm} \mathcal{C}[f]\left(x_{0}, \underline{x}\right)= \pm \frac{1}{2} \overline{e_{0}} f(\underline{x})+\frac{1}{2} H[f](\underline{x})
$$

showing formally the same structure as the Plemelj-Sokhotski formulae in the complex plane, up to the appearance of the additional factor $\overline{e_{0}}$. Here the Hilbert transform $H[f]$ of $f$ is defined as the convolution $[\mathcal{H}(\cdot) * f(\cdot)](\underline{x})$ with the Hilbert kernel

$$
\mathcal{H}(\underline{x})=-\frac{2}{a_{m+1}} \frac{\underline{x}}{|\underline{x}|^{m+1}}
$$

i.e.

$$
H[f](\underline{x})=\frac{2}{a_{m+1}} \int_{\mathbb{R}^{m}} \frac{(\underline{y}-\underline{x})}{\mid(\underline{y}-\underline{x})^{m+1}} f(\underline{y}) d \underline{y}
$$

Observe that $H^{2}=\mathbf{1}$, the identity transform. Similarly as in the complex plane, here the notion of a monogenic signal can be introduced, as the non-tangential boundary value of the monogenic Cauchy integral in the upper half space, i.e. $\frac{1}{2} \overline{e_{0}} f(\underline{x})+\frac{1}{2} H[f](\underline{x})$.

Remark 3. The Cauchy and Hilbert transforms may also be considered on the smooth boundary of a compact orientable manifold, leading to formally similar Plemelj-Sokhotski formulae as above, when taking non-tangential boundary limits. A particularly interesting case is the one of the unit sphere, studied in detail in [Brackx and De Schepper, 2008].

\subsection{The Radon transform}

The classical Radon transform is the map which assigns to a given function $f$ the totality of its integrals over all (hyper)planes of a given dimension. One of the main problems of integral geometry is to reconstruct the function $f$ from the information contained in these "sliced profiles". 
In particular, in the case of codimension one, the Radon transform is given by

$$
\mathcal{R}[f](\underline{\omega}, s)=\int_{\underline{x} \in \mathbb{R}^{m}} \delta(\langle\underline{x}, \underline{\omega}\rangle-s) f(\underline{x}) d V(\underline{x})=\int_{\langle\underline{x}, \underline{\omega}\rangle=s} f(\underline{x}) d V(\underline{x})
$$

with $\underline{\omega}$ a unit vector in $\mathbb{R}^{m}$ and $s$ a real variable. A detailed treatment of the theory of Radon transforms is to be found in the classical works [Gel'Fand et al, 1980] and [Helgason, 1980], while applications are extensively treated in [Deans, 1983] and the references therein.

As expected, the interaction between integral geometry and Clifford analysis leads to interesting observations. First notice that the above definition (5) can be taken over directly in the current Clifford framework and may still be rewritten as

$$
\mathcal{R}[f](\underline{\omega},\langle\underline{x}, \underline{\omega}\rangle)=\delta(\langle\underline{x}, \underline{\omega}\rangle) * f
$$

Alternatively, inspired by the construction of a monogenic Clifford-Radon kernel as the plane wave version of the Cauchy kernel (see Example 14), one may introduce the following CliffordRadon transform:

$$
\mathcal{C R}[f]\left(\underline{\omega},\langle\underline{x}, \underline{\omega}\rangle, x_{0}\right)=-\frac{1}{2 \pi} \int_{\mathbb{R}^{m}} \frac{\underline{\omega} f(\underline{u})}{\langle\underline{x}, \underline{\omega}\rangle-\langle\underline{u}, \underline{\omega}\rangle-x_{0} \underline{\omega}} d V(\underline{u})
$$

or still

$$
\mathcal{C R}[f]\left(\underline{\omega}, p, x_{0}\right)=-\frac{1}{2 \pi} \int_{\mathbb{R}^{m}} \frac{\underline{\omega} f(\underline{u})}{p-\langle\underline{u}, \underline{\omega}\rangle-x_{0} \underline{\omega}} d V(\underline{u})
$$

Since now the kernel function is monogenic, it directly follows that, for a chosen unit vector $\underline{\omega}$ the resulting function $\mathcal{C R}[f]\left(\underline{\omega}, p, x_{0}\right)$ is monogenic in $\mathbb{R}^{m+1} \backslash \mathbb{R}^{m}$. Moreover, taking boundary values, one obtains a remarkable relation with the classical Radon transform:

$$
\lim _{\varepsilon \rightarrow 0+} \mathcal{C} \mathcal{R}[f](\underline{\omega},\langle\underline{x}, \underline{\omega}\rangle, \varepsilon)-\mathcal{C} \mathcal{R}[f](\underline{\omega},\langle\underline{x}, \underline{\omega}\rangle,-\varepsilon)=\mathcal{R}[f](\underline{\omega},\langle\underline{x}, \underline{\omega}\rangle)
$$

More details on the Radon transform in Clifford analysis, more particularly on the interesting problem of Radon inversion, can be found in [Sommen, 1992b].

\section{Conclusions}

The basic notions and results of Clifford analysis have been introduced. An intuitive approach was chosen, illustrated with many examples, which should allow the reader to get a feeling of the particularities and richness of this, by now well established, multi-dimensional function theory, as well as of its potential for applications.

More recently, however, various new branches, refinements and generalizations of Clifford analysis have emerged, which do merit to be briefly mentioned in this introductory chapter.

Hermitian Clifford analysis is a refinement of the above described setting, in the sense that the Dirac operator is split into two Hermitian conjugate Dirac operators, leading to the study of the joint null solutions of both, called h-monogenic functions. This splitting arises in a natural way when equipping the underlying vector space with a complex structure and leads to a reduction of the invariance of the system to the unitary group, see [Brackx et al, 2007a, Brackx et al, 2007b]. The first seeds of this function theory were sown in the book by Rocha-Chavez, Shapiro and Sommen, see [Rocha-Chavez et al, 2002], and later in the papers [Sabadini and Sommen, 2002] and [Brackx et al, 2008]. It was further developed in a series of papers, see e.g. [Brackx et al, 2010, 
Brackx et al, 2011a, Brackx et al, 2011b]. Hermitian Clifford analysis is the subject of another chapter in this handbook, so the interested reader may also consult this chapter and the references therein.

Discrete Clifford analysis was introduced following the need for numerical applications. In a discrete higher dimensional setting, null solutions are studied of a deliberately introduced discrete Dirac operator. Several approaches have been followed, either starting from the applications, see e.g. [Gürlebeck and Hommel, 2002, Gürlebeck and Hommel, 2003] or from a function theoretic point of view, see e.g. [Faustino and Kähler, De Schepper et al, 2009, De Ridder et al, 2010]. In the latter contribution, a theoretical framework is developed, based on skew Weyl relations, which has allowed for the development of a true discrete counterpart of Clifford analysis; the corresponding function theory was further developed e.g. in [De Ridder et al, 2011, De Ridder et al, 2012]. Discrete Clifford analysis also is treated as an independent topic in this handbook.

A representation theoretic point of view on the Dirac operator has lead to an important generalization: higher spin Dirac systems. Here one considers the Dirac operator as a differential operator mapping spinor-valued polynomials to the same space, spinor space being considered as an irreducible representation of the Spin group. The main idea then is to replace spinor space by another irreducible representation of the Spin group, in this way generalizing the Dirac operator. The fundamental paper in this respect is [Bureš et al, 2001], treating the Rarita-Schwinger operator, which is important in theoretical physics for describing particles with half-integer spin, replacing the traditional spin $1 / 2$ of the electron. Further progress has been made in a series of papers on the construction of the higher spin Dirac operator, its properties and the description of its kernel, see e.g. [Brackx et al, 2011c, Brackx et al, 2011d, De Schepper et al, 2010], and recently also in the very important paper [Eelbode et al, 2013] where a fundamental solution for the general higher spin Dirac operator is determined, allowing for the generalization of Stokes' theorem, the Cauchy-Pompeiu theorem and Cauchy's integral formula, which lie at the very basis of the further development of the function theory behind arbitrary elliptic higher spin operators. A chapter on higher spin Dirac systems is included in this handbook as well.

Finally, Clifford and harmonic analysis on super space is a very promising domain, since it has many applications in theoretical physics. The fundamental paper here is [De Bie and Sommen, 2007], where the theoretical framework is defined, based on orthogonal and symplectic Clifford algebra generators, allowing for the canonical introduction of a super-Dirac operator, a super-Laplace operator and the like. The paper [De Bie and Sommen, 2008], dealing with the fundamental solutions of the super-operators under consideration, then enabled the further development of the corresponding function theories, see e.g. [De Bie, 2008a, Coulembier et al, 2009, Coulembier et al, 2010] and its physical applications, see e.g. [De Bie, 2008b].

\section{References}

[Bory Reyes and Shapiro, 2010] J. Bory-Reyes, M. Shapiro, Clifford analysis versus its quaternionic counterparts, Math. Meth. Appl. Sci. 33(9), 2010, 1089-1101.

[Brackx et al, 2007a] F. Brackx, J. Bureš, H. De Schepper, D. Eelbode, F. Sommen, V. Souček, Fundaments of Hermitian Clifford analysis - Part I: Complex structure, Compl. Anal. Oper. Theory 1 (3), 2007, 341-365.

[Brackx et al, 2007b] F. Brackx, J. Bureš, H. De Schepper, D. Eelbode, F. Sommen, V. Souček, Fundaments of Hermitian Clifford analysis - Part II: Splitting of $h$-monogenic equations, Complex Var. Elliptic Eq. 52 (10-11), 2007, 1063-1079. 
[Brackx et al, 1982] F. Brackx, R. Delanghe, F. Sommen, Clifford analysis, Pitman, London, 1982.

[Brackx and De Schepper, 2008] F. Brackx, H. De Schepper, The Hilbert transform on a smooth closed hypersurface, Cubo - A Math. J. 10(2), 83-106.

[Brackx et al, 2010] F. Brackx, H. De Schepper, D. Eelbode, V. Souček, The Howe dual pair in Hermitian Clifford analysis, Rev. Mat. Iberoamericana 26 (2), 2010, 449-479.

[Brackx et al, 2011a] F. Brackx, H. De Schepper, R. Lávička, V. Souček, The CauchyKovalevskaya Extension Theorem in Hermitian Clifford Analysis, J. Math. Anal. Appl. 381 (2), 2011, 649-660.

[Brackx et al, 2011b] F. Brackx, H. De Schepper, R. Lávička, V. Souček, Gel'fand-Tsetlin Bases of Orthogonal Polynomials in Hermitian Clifford Analysis, Math. Meth. Appl. Sci. 34, 2011, $2167-2180$.

[Brackx et al, 2008] F. Brackx, H. De Schepper, F. Sommen, The Hermitian Clifford analysis toolbox, Adv. Appl. Cliff. Alg. 18 (3-4), 2008, 451-487.

[Brackx et al, 2005] F. Brackx, N. De Schepper, F. Sommen, The Clifford-Fourier transform, J. Fourier Anal. Appl. 11, 2005, 669-681.

[Brackx et al, 2011c] F. Brackx, D. Eelbode, L. Van de Voorde, The polynomial null solutions of a higher spin Dirac operator in two vector variables, Adv. Appl. Cliff. Alg. 21(3), 2011, 455-476.

[Brackx et al, 2011d] F. Brackx, D. Eelbode, T. Raeymaekers, L. Van de Voorde, Triple monogenic functions and higher spin Dirac operators, Int. J. Math. 22(6), 2011, 759-774.

[Brackx and Sommen, 2000] F. Brackx, F. Sommen, Clifford-Hermite wavelets in Euclidean space, J. Fourier Anal. Appl. 6(3), 2000, 299-310.

[Brauer and Weyl, 1935] R. Brauer, H. Weyl, Spinors in $n$ dimensions, Am. J. Math. 57, 1935, $425-449$

[Bureš et al, 2001] J. Bureš, F. Sommen, V. Souček, P. Van Lancker, RaritaSchwinger type operators in Clifford analysis, J. Funct. Anal. 185, 2001, 425-456.

[Cauchy,1882] A. Cauchy, Oeuvres completes, Série 1(VII), Gauthier-Villars, Paris, 1882-1974.

[Chevalley, 1997] C. Chevalley, The algebraic theory of spinors and Clifford algebras (Collected works, Volume 2), Springer Verlag, Berlin, 1997.

[Clifford, 1882] W.K. Clifford, On the classification of geometric algebras. In: R. Tucker (ed.), Mathematical papers, MacMillan, London, 1882, 397-401.

[Colombo et al, 2004] F. Colombo, I. Sabadini, F. Sommen, D.C. Struppa, Analysis of Dirac systems and computational algebra, Progress in Mathematical Physics 39, Birkhäuser, Basel, 2004.

[Common and Sommen, 1993] A.K. Common, F. Sommen, Axial monogenic functions from holomorphic functions, J. Math. Anal. Appl. 179(2), 1993, 610-629.

[Cooke] R. Cooke, The Cauchy-Kovalevskaya Theorem (preprint, available online: http://www.cems.uvm.edu/ cooke/ckthm.pdf).

[Coulembier and De Bie, 2011] K. Coulembier, H. De Bie , Hilbert space for quantum mechanics on superspace, J. Math. Phys. 52, 2011, 063504, 30 pages. 
[Coulembier et al, 2009] K. Coulembier, H. De Bie, F. Sommen, Integration in superspace using distribution theory, J. Phys. A 42, 2009, 395206, 23 pages.

[Coulembier et al, 2010] K. Coulembier, H. De Bie, F. Sommen, Orthosymplectically invariant functions in superspace, J. Math. Phys. 51, 2010, 083504, 23 pages.

[Deans, 1983] S. R. Deans, The Radon Transform and some of its applications, Wiley-Interscience, New York, 1983.

[De Bie, 2008a] H. De Bie, Fourier transform and related integral transforms in superspace, $J$. Math. Anal. Appl. 345, 2008, 147-164.

[De Bie, 2008b] H. De Bie, Schrödinger equation with delta potential in superspace, Phys. Lett. A 372, 2008, 4350-4352.

[De Bie and Sommen, 2007] H. De Bie, F. Sommen, A Clifford analysis approach to superspace, Ann. Phys. 322(12), 2007, 2978-2993.

[De Bie and Sommen, 2008] H. De Bie, F. Sommen, Fundamental solutions for the super Laplace and Dirac operators and all their natural powers, J. Math. Anal. Appl. 338, 2008, 1320-1328.

[Delanghe,1970] R. Delanghe, On regular-analytic functions with values in a Clifford algebra, Math. Ann. 185, 1970, 91-111.

[Delanghe, 2001] R. Delanghe, Clifford analysis: History and perspective, Comp. Meth. Funct. Theo. 1, 2001, 107-153.

[Delanghe et al, 1992] R. Delanghe, F. Sommen, V. Souček, Clifford algebra and spinor-valued functions - A function theory for the Dirac operator, Kluwer Academic Publ., Dordrecht, 1992.

[De Ridder et al, 2010] H. De Ridder, H. De Schepper, U. Kähler, F. Sommen, Discrete function theory based on skew Weyl relations, Proc. Amer. Math. Soc. 138, 2010, 3241-3256.

[De Ridder et al, 2011] H. De Ridder, H. De Schepper, F. Sommen, The Cauchy-Kovalevskaya Extension Theorem in Discrete Clifford Analysis, Comm. Pure Appl. Math 10(4), 2011, 10971109.

[De Ridder et al, 2012] H. De Ridder, H. De Schepper, F. Sommen, Fueter polynomials in discrete Clifford analysis, Math. Zeit. 272(1-2), 2012, 253-268.

[De Schepper et al, 2010] H. De Schepper, D. Eelbode, T. Raeymaekers, On a special type of solutions of arbitrary higher spin Dirac operators, J. Phys. A 43(32) ,2010), 1-13.

[De Schepper et al, 2009] H. De Schepper, F. Sommen, L. Van de Voorde, A basic framework for discrete Clifford analysis, Experimental Mathematics 18(4), 2009, 385-395.

[De Schepper and Sommen, 2012] N. De Schepper, F. Sommen, Cauchy-Kowalevski extensions and monogenic plane waves in Clifford analysis, Adv. Appl. Cliff. Alg. 22(3), 2012, 625-647.

[Dirac, 1928] P.A.M. Dirac, The quantum theory of the electron I-II, Proc. Roy. Soc. London A117, 1928, 610-524; A118, 1928, 351-361.

[Eelbode et al, 2013] D. Eelbode, T. Raeymaekers, P. Van Lancker, On the fundamental solution for higher spin Dirac operators, J. Math. Anal. Appl. 405(2), 2013, 555-564. 
[Faustino and Kähler] N. Faustino, U. Kähler, Fischer decomposition for difference Dirac operators, Adv. Appl. Cliff. Alg. 17(1), 2007, 37-58.

[Fueter, 1935a] R. Fueter, Zur Theorie der regulären Funktionen einer Quaternionenvariablen, Monat. für Math. und Phys. 43, 1935, 69-74.

[Fueter,1935b] R. Fueter, Die funktionentheorie der differentialgleichungen $\Delta u=0$ und $\Delta \Delta u=0$ mit vier variablen, Comm. Math. Helv. 7, 1935, 307-330.

[Gel'Fand et al, 1980] I.M. Gel'Fand, S.G. Gindikin, M.I. Graev, Integral geometry in affine and projective spaces, J. Sov. Math 18, 1980, 39-67.

[Gilbert and Murray, 1991] J. Gilbert, M. Murray, Clifford Algebra and Dirac Operators in Harmonic Analysis, Cambridge University Press, Cambridge, 1991.

[Gürlebeck et al, 2008] K. Gürlebeck, K. Habetha, W. Sprößig, Holomorphic functions in the plane and n-dimensional space, Birkhäuser Verlag, Basel, 2008.

[Gürlebeck and Hommel, 2002] K. Gürlebeck, A. Hommel, On finite difference potentials and their applications in a discrete function theory, Math. Meth. Appl. Sci. 25, 2002, 1563-1576.

[Gürlebeck and Hommel, 2003] K. Gürlebeck, A. Hommel, On finite difference Dirac operators and their fundamental solutions, Adv. Appl. Cliff. Alg. 11, 2003, 89-106.

[Gürlebeck and Sprössig] K. Gürlebeck, W. Sprößig, Quaternionic and Clifford Calculus for Physicists and Engineers, J. Wiley \& Sons, Chichester, 1997.

[Hahn, 1996] S.L. Hahn, Hilbert transforms in signal processing, Artech House, Boston, 1996.

[Helgason, 1980] S. Helgason, The Radon Transform, Birkhäuser, Boston, 1980.

[Kowalevsky,1875] S. Kowalevsky, Zur Theorie der partiellen Differentialgleichung, J. für die Reine und Angew. Mathem. 80, 1875, 1-32.

[Li et al, 1994] C. Li, A. Mc Intosh, T. Qian, Fourier transforms and singular convolution operators on Lipschitz surfaces, Rev. Math. IberoAmer. 10, 1994, 665-721.

[Qian, 1997] T. Qian, Generalization of Fueter's result to $\mathbb{R}^{n+1}$, Rend. Mat. Acc. Lincei 8, 1997, $111-117$.

[Rocha-Chavez et al, 2002] R. Rocha-Chavez, M. Shapiro, F. Sommen, Integral theorems for functions and differential forms in $\mathbb{C}_{m}$, Research Notes in Math. 428, Chapman\&Hall / CRC, New York, 2002.

[Ryan,2000] J. Ryan, Basic Clifford Analysis, Cubo Math. Educ. 2, 2000, 226-256.

[Sabadini and Sommen, 2002] I. Sabadini, F. Sommen, Hermitian Clifford analysis and resolutions, Math. Meth. Appl. Sci. 25(16-18), 2002, 1395-1414.

[Sce,1957] M. Sce, Osservazioni sulle serie di potenze nei moduli quadratici, Atti Accad. Naz. Lincei. Rend. Cl. Sci. Fis. Mat. Nat. (8) 23, 1957, 220-225.

[Sommen, 1988] F. Sommen, Plane wave decompositions of monogenic functions, Annales Pol. Math. 49, 1988, 101-114.

[Sommen, 1992a] F. Sommen, Clifford analysis and integral geometry. In: Proc. Second Workshop on Clifford Algebras, Kluwer Acad. Publ., 1992, 293-311. 
[Sommen, 1992b] F. Sommen, Clifford analysis and integral geometry. In: A. Micali et al. (eds.), Clifford algebras and their applications in mathematical physics, Fund. Theories Phys. 47, Kluwer Academic Publishers, Dordrecht, 1992, 293-311.

[Stein,1970] E. Stein, Singular integrals and differentiability properties of functions, Princeton University Press, Princeton, 1970. 